\title{
Obesidade, inflamação e exercício: foco sobre o TNF-alfa e IL-10
}

\author{
Obesity, inflammation and exercise: focus on TNF-alpha and IL-10
}

\author{
Guilherme Fleury Fina Speretta*, Richard Diego Leite, Ana Cláudia Garcia de Oliveira Duarte
}

\begin{abstract}
Resumo
O aumento da prevalência da obesidade está diretamente relacionado ao aumento da ingestão calórica associado à diminuição do gasto energético, devido às alterações no estilo de vida do ser humano. O agravante dessa situação é que doenças crônico-degenerativas como diabetes mellitus tipo 2, hipertensão arterial, aterosclerose, esteatose hepática não alcoólica que, em conjunto, caracterizam a síndrome metabólica, estão acometendo mais pessoas a cada dia, independentemente de classe social, idade e gênero. Apesar de ainda não existir um consenso sobre a etiologia da síndrome metabólica, parece estar claro na literatura que a inflamação crônica de baixa intensidade, originada a partir do excesso de tecido adiposo, é um fator que está presente na maioria das doenças que compõem essa síndrome. Diversos estudos têm demonstrado que a hipertrofia dos adipócitos, principalmente no tecido adiposo visceral, provoca um desequilíbrio na homeostase metabólica do tecido adiposo, aumentando a produção de adipocinas pró-inflamatórias, como o fator de necrose tumoral-alfa (tumor necrosis factor alpha - TNF-alfa) e reduzindo a produção de adipocinas anti-inflamatórias, como interleucina-10 (IL-10), culminando no processo inflamatório crônico de baixa intensidade. Dessa maneira, diferentes modalidades de exercício físico têm se mostrado estratégias capazes de promover inúmeros benefícios na prevenção e no tratamento da obesidade, inclusive na redução do processo inflamatório crônico de baixa intensidade ou, em outras palavras, diminuindo a produção de TNF-alfa e outras adipocinas pró-inflamatórias e aumentando a produção de IL-10 e outras adipocinas anti-inflamatórias.
\end{abstract}

Descritores: Obesidade; Inflamação; Exercício.

\begin{abstract}
The increasing prevalence of obesity is directly related to increased caloric intake associated with decreased energy expenditure, due to changes in lifestyle of humans. To aggravate this situation, chronic diseases such as type 2 diabetes mellitus, hypertension, atherosclerosis, nonalcoholic fatty liver disease, which together characterize the metabolic syndrome, are affecting more people every day, regardless of social class, age and gender. Although there is no consensus on the etiology of the metabolic syndrome, it seems in the literature that low-grade chronic inflammation caused by excess fatty tissue is a factor that is present in most of the diseases that make up this syndrome. Several studies have shown that adipocyte hypertrophy, especially of visceral adipose tissue, causes an imbalance in metabolic homeostasis of adipose tissue, increasing the production of proinflammatory adipokines, such as tumor necrosis factor-alpha (TNF-alfa) and reducing the production of anti-inflammatory adipokines, such as interleukin-10 (IL-10), culminating in the chronic low-grade inflammatory process. Thus, different modalities of exercise have been shown as strategies that may promote many benefits in the prevention and treatment of obesity, including reduction in low-grade chronic inflammation, or in other words, decreasing the production of TNF-alfa and other proinflammatory adipokines and increasing the production of IL-10 and other anti-inflammatory adipokines.
\end{abstract}

Keywords: Obesity; Inflammation; Exercise. 


\section{Introdução}

Nas últimas décadas, a prevalência de obesidade tem aumentado em todo o mundo. Dados recentes estimam que aproximadamente 500 milhões de pessoas adultas (acima de 20 anos) sejam obesas no mundo, das quais 205 milhões são homens $(9,8 \%)$ e 297 milhões são mulheres (13,8\%). ${ }^{1}$ No Brasil, segundo dados divulgados pelo Instituto Brasileiro de Geografia e Estatística (IBGE), ${ }^{2}$ a incidência de obesidade é de $12,4 \%$ e $16,9 \%$ em homens e mulheres, respectivamente.

A obesidade foi definida pela Organização Mundial de Saúde, em 2000, como o acúmulo anormal ou excessivo de gordura [índice de massa corporal $(\mathrm{IMC}) \geq 30 \mathrm{~kg} / \mathrm{m}^{2}$ ] que representa risco à saúde. ${ }^{3}$ Contudo, o aumento exponencial na prevalência da obesidade e o consequente interesse dos pesquisadores em estudar essa doença permitiram a ampliação desse conceito. Hoje, essa enfermidade pode ser definida como de origem multifatorial, resultante de fatores genéticos, fisiológicos, ambientais e psicológicos, proporcionando o acúmulo excessivo de energia sob a forma de gordura no organismo. ${ }^{4,5}$

Recentes investigações associam a obesidade a outras doenças crônico-degenerativas, tais como dislipidemia, hiperinsulinemia, diabetes mellitus tipo 2 (DM2), hipertensão arterial, aterosclerose e, mais recentemente, a esteatose hepática não alcoólica e alguns tipos de câncer, que, em conjunto, contribuem para o aumento da morbimortalidade em todo o mundo. Essas alterações, com exceção do câncer, coletivamente, compreendem o diagnóstico da síndrome metabólica (SM). Assim como a obesidade, a SM tem sua etiologia associada a fatores genéticos e ambientais. Além disso, sua prevalência também vem crescendo de forma alarmante. ${ }^{6,7}$

Gerald Reaven ${ }^{8}$ foi o primeiro autor a descrever a SM. Na oportunidade, o autor propôs que a resistência à ação da insulina seria o alvo central da etiologia do DM2, da doença coronariana e da hipertensão arterial. No entanto, evidências mais recentes apontam para uma relação muito próxima entre SM e obesidade, especialmente a "obesidade visceral". 9

Nesse contexto, uma revisão ${ }^{10}$ apontou diversos biomarcadores comuns entre obesidade e SM: IMC (obesidade), circunferência de cintura (obesidade abdominal), área e volume dos adipócitos viscerais (obesidade visceral), gordura hepática (gordura ectópica), leptina (desordens endócrinas), fator de necrose tumoral-alfa (TNF-alfa - inflamação) e acúmulo de macrófagos (modificações histológicas). Além disso, a obesidade visceral associada a fatores genéticos e ambientais, como fumo e estresse, é componente essencial na fisiopatologia da SM. ${ }^{9}$ Em contrapartida, outros achados afirmam que ainda não está claro qual é o principal desencadeador da $\mathrm{SM}^{11} \mathrm{e}$ que não existe um consenso sobre sua definição, o que coloca em risco seu diagnóstico. ${ }^{2}$

O real impacto do exercício como estratégia não farmacológica de tratamento da obesidade ainda merece esclarecimento, principalmente em relação aos seus efeitos sobre citocinas pró-inflamatórias, como o TNF-alfa e a interleucina-10 (IL10). Desse modo, o presente trabalho tem como objetivo demonstrar e discutir a relação entre obesidade e inflamação, com foco principal no TNF-alfa e IL-10, bem como demonstrar os efeitos do exercício sobre estas citocinas.

Para tanto, foi realizada uma revisão de literatura, através de busca científica on-line, de caráter descritivo e exploratório, a partir de trabalhos relacionados à obesidade, inflamação e exercício, com foco em TNF-alfa e lL-10. Foram consultadas as bases de dados PubMed, MEDLINE, SciELO, LILACS, Periódicos Portal Capes, Highwire e Isi Web of Knowledge. Uma análise semiquantitativa do material encontrado na literatura foi realizada, baseada nos principais focos do trabalho.

\section{Obesidade e inflamação}

Durante décadas, o tecido adiposo foi considerado como um órgão com papel crucial apenas na regulação da homeostase dos ácidos graxos do organismo. Em períodos de abundância de calorias, os ácidos graxos livres são armazenados na forma de triacilglicerol através da sua esterificação com glicerol e, em tempos de escassez de energia, estes são liberados de volta para a circulação. ${ }^{13}$

Foi com a descoberta da leptina, hormônio regulador da ingestão de alimentos e do balanço energético, e a confirmação da secreção de proteínas envolvidas na regulação do metabolismo, como o TNF-alfa, identificada como um regulador 
negativo da transdução do sinal de insulina, que o tecido adiposo branco passou a ser considerado um órgão secretor. ${ }^{14}$ Sabe-se hoje que o tecido adiposo possui, além de capacidade de regulação do armazenamento e da distribuição de gordura, comunicação com o sistema nervoso central e o trato gastrintestinal, desempenhando importante papel na resposta inflamatória em condições tanto autócrinas quanto parácrinas ou endócrinas. ${ }^{15}$

Atualmente, mais de cinquenta produtos derivados dos adipócitos já foram isolados e caracterizados. ${ }^{16}$ Nesse sentido, estudos experimentais mostraram que ratos obesos aumentam não apenas os níveis de TNF-alfa, mas também de outras adipocinas pró-inflamatórias incluindo as interleucinas 6 e 1-beta, quimiocina ligante CC 2 (CCL2), entre outras. ${ }^{14}$ Em contrapartida, ratos obesos diminuem os níveis de adipocinas anti-inflamatórias, como a IL-10. ${ }^{17}$

Segundo Heber, ${ }^{15}$ a localização da gordura corporal também tem implicações significativas para os riscos à saúde, sendo que riscos maiores estão associados à obesidade abdominal. Este aumento é infindo, podendo ultrapassar a capacidade de suprimento de sangue para estas células, com a possibilidade de interação com monócitos atraídos a partir da medula óssea.

Estudos recentes mostram que a hipertrofia dos adipócitos, isto é, o aumento do volume da célula adiposa, decorrente do acúmulo excessivo de triacilgliceróis, é altamente correlata com a obesidade e acarreta uma infiltração e ativação de macrófagos no tecido adiposo. Isso, por sua vez, culmina no aumento do processo inflamatório crônico de baixa intensidade..$^{13-15}$

A hipertrofia dos adipócitos, especialmente os viscerais, que são mais ativos, está relacionada com a resistência ao efeito antilipolítico da insulina e aumento da ação das catecolaminas. Isso se deve ao aumento da expressão de beta-adrenoreceptores (beta-3) e à diminuição da expressão de alfa-2-adrenoreceptor, aumentando o fluxo dos ácidos graxos não esterificados para o fígado, via sistema porta, culminando em maior produção de glicose hepática, redução da degradação de apolipoproteína B e aumento da produção de triacilgliceróis. Todas essas alterações descritas (alteração nos mecanismos de síntese e oxidação de lipídios, bem como sua função secretória) são fatores que indicam o envolvimento do tecido adiposo com a fisiopatologia da SM. ${ }^{9}$
A hipertrofia dos adipócitos induz à infiltração de macrófagos e ao aumento da inflamação com produção aumentada de adipocinas pró-inflamatórias, como TNF-alfa e IL-6. Isso é acompanhado por um aumento da liberação de ácidos graxos livres e desregulação da secreção de leptina, adiponectina, resistina e proteína ligante de retinol (RBP4). Juntas, essas substâncias derivadas dos adipócitos e macrófagos podem agir de forma parácrina ou autócrina, agravando a inflamação do tecido adiposo. Em nível sistêmico, a secreção alterada de adipocinas pode levar ao aumento na ingestão alimentar e à redução do gasto energético através de ações no hipotálamo. Além disso, a sensibilidade à insulina diminui no músculo e no fígado através do aumento ectópico da deposição de lipídios associado ao desenvolvimento da inflamação crônica de baixa intensidade ${ }^{13} \mathrm{O}$ resumo destes mecanismos está ilustrado na figura 1.

\section{TNF-alfa}

TNF-alfa é uma citocina pró-inflamatória, ${ }^{17}$ sintetizada como uma proteína transmembrana com massa molecular de $26 \mathrm{kDa}$. Ela passa por uma clivagem antes de ser liberada para a circulação como uma molécula solúvel. ${ }^{13}$ Estudos indicam que a hipertrofia do tecido adiposo leva ao aumento da expressão de TNF-alfa, tendo ações predominantemente autócrinas e parácrinas. ${ }^{14}$

Originalmente pensava-se que a razão para o aumento observado dos níveis séricos desta citocina em indivíduos obesos fosse a superprodução realizada pelo excesso de tecido adiposo. Entretanto, atualmente tem sido reconhecido que esse aumento é devido à infiltração de macrófagos M1 no tecido adiposo. ${ }^{13,15,17}$ Independente do agente responsável pelo aumento de TNF-alfa, inúmeros achados demonstram que níveis elevados desta citocina estão relacionados com a resistência à insulina ${ }^{17} \mathrm{e}$, consequentemente, com a etiologia do DM2.11,13,14,18

O TNF-alfa parece ter papel importante na fisiopatologia da resistência à insulina através da diminuição da expressão à superfície celular dos transportadores de glicose (GLUT-4), fosforilação do substrato 1 dos receptores de insulina (IRS-1) e fosforilação específica do receptor da insulina. ${ }^{17}$

Galic e colaboradores, ${ }^{13}$ em uma revisão de literatura, ampliaram a gama de mecanismos que 
envolvem o TNF-alfa, com a resistência à ação da insulina incluindo bases moleculares como a inibição da capacidade de sinalização da IRS-1 através da ativação de serinoquinases, tais como a quinase c-Jun $\mathrm{N}$-terminal (JNK) ou complexo quinase lkB (lkK), além de aumentar a expressão de citocinas supressoras da sinalização 3 (SOCS3). O TNF-alfa também reduz a oxidação de ácidos graxos em hepatócitos e músculos esqueléticos por meio de efeitos mediados pela indução da pro- teína fosfatase $2 \mathrm{C}$ e supressão da quinase ativada por AMP (AMPK). As taxas reduzidas de oxidação dos ácidos graxos são acompanhadas por acúmulo de lipídios bioativos, como triacilgliceróis, que por sua vez, são conhecidas por ativar a proteína quinase $\mathrm{C}$ e inibir a função do IRS.

Uma investigação recente confirmou que uma dieta hiperlipídica aumenta a expressão de TNF-alfa, lkK e outros fatores que caracterizam o aumento do processo inflamatório no tecido

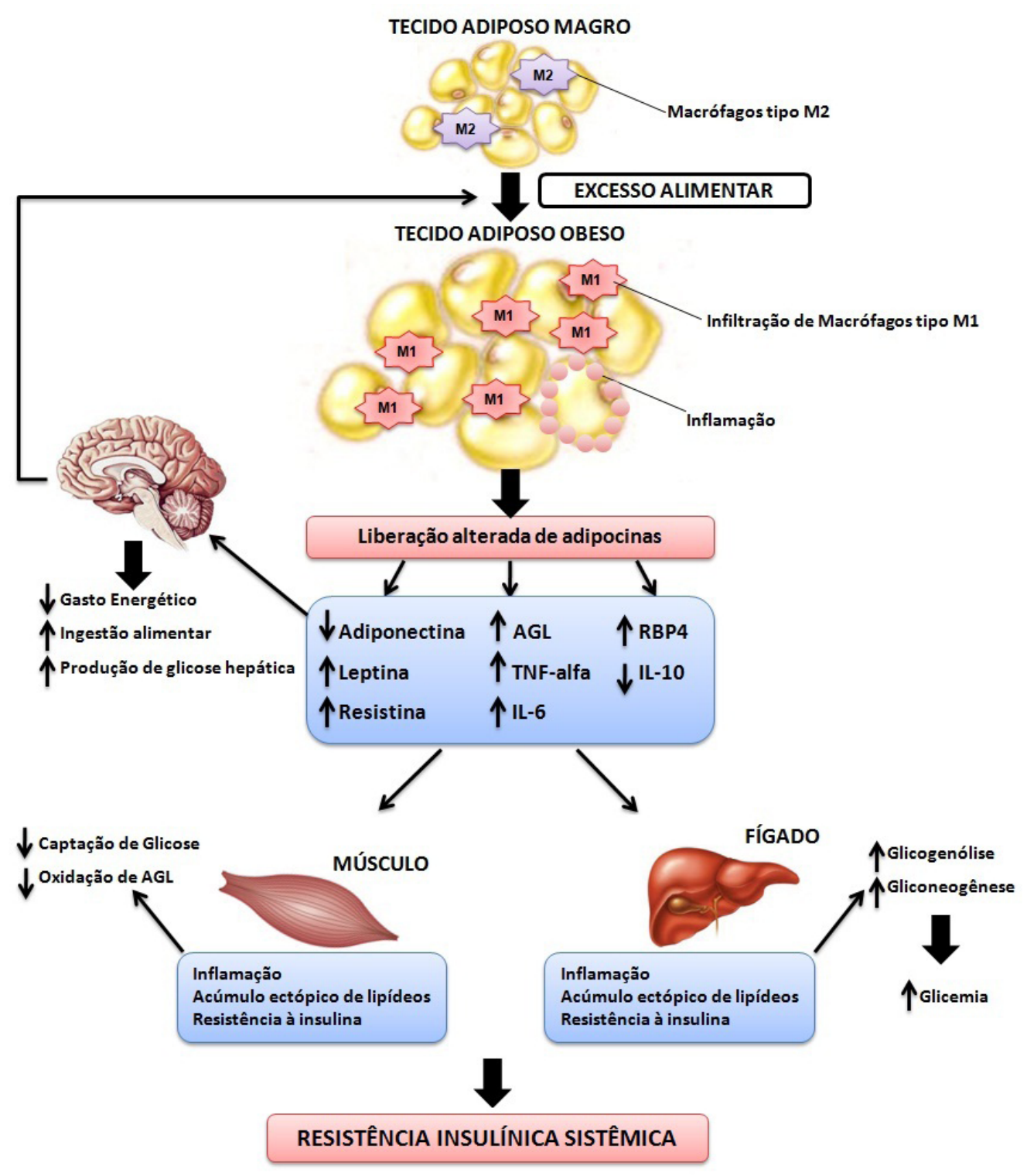

Figura 1. Hipertrofia do tecido adiposo induz a infiltração e proliferação de macrófagos e alteração na secreção de adipocinas levando à inflamação crônica de baixa intensidade. Este quadro, associado ao aumento de ácidos graxos livres circulantes, provoca aumento de ingestão alimentar, diminuição do gasto energético, além da alteração na homeostase de tecidos periféricos, como músculo e fígado, promovendo acúmulo ectópico de gordura, inflamação e resistência à insulina.

Fonte: Adaptada de Galic e colaboradores. ${ }^{13}$ 
adiposo de camundongos. Além disso, demonstra também que o aumento no processo inflamatório está associado a aumentos concomitantes nos níveis de insulina, leptina e glicose, reforçando a relação do processo inflamatório crônico de baixa intensidade com a resistência insulínica. ${ }^{19} \mathrm{Em}$ humanos também demonstrou-se que o excesso de peso (IMC $>27 \mathrm{~kg} / \mathrm{m}^{2}$ ) contribui para o aumento dos níveis séricos de TNF-alfa, apresentando correlação inversa entre o aumento desta citocina e o metabolismo da glicose..$^{20}$

Adicionalmente, o TNF-alfa parece ativar o fator de transcrição nuclear kB (NF-kB), o que leva a uma série de alterações inflamatórias no tecido vascular. Essas alterações inflamatórias do tecido vascular resulta em ativação endotelial, com disfunção endotelial e hipertensão. ${ }^{21}$ Entretanto, diversos estudos demonstraram que a redução de peso e a redução de gordura visceral, especificamente, pode aumentar os níveis de adipocinas anti-inflamatórias, como adiponectina e IL-10, e reduzir os níveis de adipocinas pró-inflamatórias, como TNF-alfa, resistina e IL-6. 13,17-19,22

\section{IL-10}

A IL-10 é uma citocina anti-inflamatória, com massa molecular de $17 \mathrm{kDa}$ em sua forma solúvel, sendo produzida principalmente por macrófagos e linfócitos. ${ }^{17}$ Evidências apontam que no tecido adiposo a IL-10 é produzida por macrófagos M2, principalmente em indivíduos magros. ${ }^{13}$ Sua principal função parece ser a regulação do sistema imune, inibindo significativamente a expressão e/ ou síntese de citocinas ou adipocinas pró-inflamatórias por meio de contrarregulação negativa. ${ }^{11,23}$

Segundo Nishida e colaboradores, ${ }^{24}$ a IL-10 tem propriedades anti-inflamatórias multifacetadas, incluindo a inibição da atividade de macrófagos e células T, além de apresentar um efeito protetor contra aterogênese. Foi demonstrado claramente na literatura que a adiponectina, uma importante adipocina anti-inflamatória, induz a expressão de IL-10 em macrófagos humanos. Aparentemente, parte dos efeitos anti-aterogênicos da adiponectina são mediados pela IL-10.1,23,24

A diminuição da concentração plasmática de IL-10 tem sido correlacionada positivamente com uma diminuição da fração de ejeção do ventrículo esquerdo. Além disso, em animais com insuficiên- cia cardíaca pós-infarto do miocárdio, a taxa de produção de IL-10 foi recentemente demonstrada como um indicador preciso do grau de disfunção ventricular. ${ }^{25}$

Recentemente, Ropelle e colaboradores ${ }^{26}$ demonstraram que a IL-10 pode exercer importante ação anti-inflamatória no sistema nervoso central. Os achados destes autores sugerem que a infusão de IL-10 diretamente no hipotálamo inibe a ação inflamatória do lkKB/NF-kB nessa região do cérebro de ratos obesos, aumentando a sensibilidade à ação da leptina e da insulina nos neurônios dessa região, culminando em diminuição da ingestão calórica e melhora no controle do balanço energético.

\section{TNF-alfa e IL-10: efeitos do exercício}

Estudos epidemiológicos e de coorte têm demonstrado forte associação entre obesidade e inatividade física, bem como tem sido descrita a associação inversa entre atividade física, índice de massa corpórea (IMC), razão cintura-quadril (RCQ) e circunferência de cintura. ${ }^{27}$

A recomendação do American College of Sports Medicine é que indivíduos que desejam manter sua massa corporal estável devem realizar de 150 a 200 minutos de exercício físico por semana, gerando um gasto calórico em torno de 1.200 a 2.000 kcal por semana. Já indivíduos que estão com sobrepeso ou são obesos devem realizar de 250 a 300 minutos de exercício físico por semana, com gasto calórico estimado de no mínimo 2.000 kcal por semana para obter uma perda de peso significativa. ${ }^{28}$ No entanto, é importante ressaltar que para um emagrecimento significativo e duradouro é essencial a associação do exercício físico com uma dieta hipocalórica (500-1.000 kcal/dia). Portanto, parece que somente o gasto calórico advindo do exercício físico não é suficiente para o sucesso de um programa de intervenção para tratar a obesidade. ${ }^{27,28}$

Inúmeras evidências têm apontado o exercício físico como importante agente estimulador de respostas anti-inflamatórias, podendo auxiliar no controle da inflamação crônica de baixa intensidade e, consequentemente, diminuírem os riscos das doenças crônicas associadas, como DM2, 
hipertensão e aterosclerose. ${ }^{18,19,22,29,30}$

Petersen e Pedersen, ${ }^{22}$ em uma revisão de literatura, afirmaram que o exercício físico regular pode proteger contra doenças associadas à inflamação crônica de baixa intensidade. Esse efeito crônico do exercício pode ser atribuído à resposta anti-inflamatória induzida pelo efeito repetido de uma sessão aguda de exercício, que é parcialmente mediada pela IL- 6 derivada do músculo esquelético. Essa citocina tem características dúbias, podendo ser pró ou anti-inflamatória. Concentrações fisiológicas de IL-6 parecem estimular o aparecimento na circulação das citocinas anti-inflamatórias: receptor antagonista de IL-1 (IL-1ra) e IL-10, além de inibir a produção de citocinas pró-inflamatórias como TNF-alfa, podendo reduzir seus efeitos deletérios. Além disso, a IL-6 parece estimular a lipólise, bem como a oxidação das gorduras.

Segundo Pauli e colaboradores, ${ }^{31}$ os benefícios do exercício são claros frente aos efeitos deletérios do processo inflamatório no músculo esquelético. Como foi dito anteriormente, o excesso de ácidos graxos livres associados ao processo inflamatório crônico de baixa intensidade, com aumento de TNF-alfa, entre outros marcadores pró-inflamatórios com IL-1-beta, podem desencadear a resistência à ação da insulina no músculo esquelético. Resumidamente, os mecanismos que explicam este quadro são a ativação de quinases, especialmente IkKB e JNK. A lkKB pode interferir na sinalização de insulina através de pelo menos duas vias: primeiro, fosforilando diretamente os substratos do receptor de insulina (IRS-1 e IRS-2) em resíduos de serina; segundo, ativando indiretamente o NF-kB, um fator de transcrição que, entre outros alvos, pode estimular a produção de vários mediadores inflamatórios, incluindo o TNF-alfa e a sintase de óxido nítrico induzida (inducible nitric oxide synthase - iNOS). Já a JNK também pode interferir negativamente na sinalização da insulina, fosforilando o IRS-1 e o IRS-2 em serina. O exercício físico parece agir reduzindo a expressão e/ou atividade destas proteínas intracelulares de efeito negativo sobre a via de sinalização da insulina, aumentando a sensibilidade à insulina e melhorando a captação de glicose pelo músculo esquelético.

Assim, Bradley e colaboradores, ${ }^{19}$ utilizando camundongos induzidos à obesidade com quatro semanas de dieta hiperlipídica, analisaram o efeito de seis semanas de corrida voluntária na expressão de: TNF-alfa, proteína quimiotática de monócitos-1 (monocyte chemoattractant protein-1 - MCP-1), inibidor do ativador do plasminogênio tipo 1 (plasminogen activator inhibitor-1 - PAl-1) e IkK no tecido adiposo visceral, a fim de verificar a homeostase glicêmica nesses animais. Os resultados desse estudo sugerem que o exercício reduz parcialmente a adiposidade, reverte a resistência à insulina e diminui a inflamação do tecido adiposo visceral, induzida pela dieta hiperlipídica.

Recentemente uma robusta investigação ${ }^{26}$ analisou o efeito agudo de uma sessão de exercício de natação ou de corrida na resistência à ação da leptina e da insulina no hipotálamo em ratos Wistar induzidos à obesidade através de três meses de ingestão de uma dieta hiperlipídica. De maneira interessante, os achados dessa investigação apontaram para uma diminuição da ingestão alimentar após uma única sessão de ambas as modalidades utilizadas, sugerindo um aumento da sensibilidade à leptina e à insulina no hipotálamo. Averiguando as razões para tal resposta, os autores observaram que o exercício promove um aumento das citocinas anti-inflamatórias no hipotálamo, como as ILs 6 e 10, induzindo à redução da atividade dos marcadores IKKb/NF-kB e do estresse no retículo endoplasmático no hipotálamo. Nesse sentido, parece que o exercício pode ser importante no combate à obesidade, não apenas na redução da massa corporal e da adiposidade, mas também na redução da ingestão alimentar induzida por uma resposta anti-inflamatória.

Batista e colaboradores ${ }^{25}$ apontam que o exercício físico, principalmente o aeróbio, também tem sido considerado o alicerce dos programas de reabilitação cardíaca e uma importante forma de tratamento não farmacológico. Segundo os autores, a IL-10 parece ser afetada pelo exercício físico, podendo atuar como fator central na atenuação e/ou modulação da resposta inflamatória na insuficiência cardíaca. Além do aumento local na produção de IL-10, esse efeito poderia ter impacto sistêmico, reduzindo, ou até mesmo evitando, o aumento de citocinas pró-inflamatórias, como TNF-alfa, condição que, no caso da insuficiência cardíaca está relacionada à maior severidade dessa doença.

Em contrapartida, um estudo de Lira e colaboradores $^{32}$ demonstrou que a falta de um tem- 
po adequado de recuperação entre sessões de exercício físico pode ser prejudicial. Os autores realizaram um protocolo progressivo de exercício em esteira com ratos Wistar, durante 11 semanas, no qual os animais aumentaram gradativamente a intensidade e o volume de exercício ao longo das semanas de treinamento, até chegarem a uma intensidade de $25 \mathrm{~m} \cdot \mathrm{min}^{-1}$ durante 60 minutos na quarta semana. A partir desse momento, a fim de criar um desequilíbrio entre estímulo e recuperação, os períodos de recuperação foram diminuídos gradativamente, passando de 24 horas na oitava semana de treinamento para 4 horas na nona, 3 horas na décima e apenas 2 horas na última semana de treinamento. Os resultados dessa investigação apontaram que o excesso de exercício aeróbio, realizado em esteira, pode contribuir para o aumento das concentrações séricas de TNF-alfa e IL-6, bem como promover o aumento na expressão de outros fatores pró-inflamatórios no tecido adiposo, como receptor do tipo Toll-4 (TLR-4) e NF-kB. Além disso, essa investigação demonstrou que os animais que treinaram de forma excessiva apresentaram concentrações séricas menores de IL-10 quando comparados àqueles que tiveram um período de recuperação adequado entre as sessões de exercício. Em suma, os autores sugerem que o "supertreinamento" pode promover um aumento do processo inflamatório crônico de baixa intensidade, mesmo em animais que não foram induzidos à obesidade.

Tendo em vista os fatos apresentados até aqui, é possível sugerir que o exercício físico aeróbio, aplicado de maneira coerente, realmente contribui de forma significativa na melhora do quadro inflamatório crônico de baixa intensidade. No entanto, o efeito direto do treinamento de força na inflamação do tecido adiposo em animais obesos permanece obscuro. Os únicos achados na literatura que utilizaram protocolos de treinamento de força $\mathrm{a}^{30,33,34}$ têm demonstrado que ele parece ser eficaz na diminuição dos depósitos de tecido adiposo em ratos.

Nesse sentido, nosso grupo de pesquisa realizou um estudo ${ }^{35}$ com ratos Wistar adultos induzidos à obesidade com três semanas de dieta hiperlipídica, com o intuito de analisar os efeitos de oito semanas de treinamento de força de alta intensidade/curta duração e do treinamento aeró- bio de moderada intensidade/longa duração sobre a expressão gênica de TNF-alfa e IL-10, área de adipócitos e perfil lipídico. Os animais dos grupos de treinamento de força realizaram escaladas em uma escada vertical, com pesos atados às suas caudas. As sessões foram realizadas uma vez a cada três dias, com 4 a 9 escaladas com 8 a 12 movimentos dinâmicos por escalada. Os grupos de natação realizaram $60 \mathrm{~min} /$ dia, cinco dias por semana, com uma carga constante de peso de $5 \%$. Os animais treinados em força apresentaram menores valores de área de adipócitos nos tecidos visceral e retroperitoneal, menor expressão de TNF-alfa no tecido adiposo visceral, expressão não alterada de IL-10 no mesmo tecido e benefícios no perfil lipídico. Os grupos de natação apresentaram menor área de adipócitos nos tecidos epididimal e retroperitoneal, menor expressão de TNF-alfa maior expressão de IL-10 e benefícios no perfil lipídico. Os resultados do nosso estudo indicam os potenciais benefícios do treinamento de força e da natação como alternativas não farmacológicas para controlar os efeitos deletérios da dieta hiperlipídica em ratos.

\section{Conclusões}

O exercício físico parece contribuir de maneira significativa na redução da atividade de importantes marcadores pró-inflamatórios, como o TNF-alfa, MCP-1, PAl-1, lkK, NF-kB, entre outros, e no aumento da atividade de marcadores anti-inflamatórios, principalmente adiponectina e IL-10.

Entretanto, em relação à diminuição do tecido adiposo e da inflamação crônica de baixa intensidade, ainda não está claro qual é a melhor modalidade de exercício, o melhor equilíbrio entre volume e intensidade, além da melhor frequência de exercício para a obtenção de melhores resultados.

\section{Agradecimentos}

Agradecemos pelo apoio financeiro, fornecido pelo Conselho Nacional de Desenvolvimento Científico e Tecnológico (CNPq), Brasil.

\section{Referências}

1. Finucane MM, Stevens GA, Cowan MJ, Danaei G, Lin JK, Paciorek CJ, et al. National, regional, and global 
trends in body-mass index since 1980: systematic analysis of health examination surveys and epidemiological studies with 960 country-years and 9.1 million participants. Lancet. 2011 Feb;377(9765):557-67. http://dx.doi.org/S0140-6736(10)62037-5 [pii]10.1016/ S0140-6736(10)62037-5

2. Instituto Brasileiro de Geografia e Estatística (IBGE). Pesquisa de Orçamentos Familiares (POF) 2008-2009 - Antropometria e estado nutricional de crianças, adolescentes e adultos no Brasil. Rio de Janeiro: IBGE; 2010 [citado em 02 fev. 2011. Available from: http://www.ibge.gov.br/home/presidencia/noticias/noticia_visualiza.php?id_noticia=1699\&id_pagina $=1$.

3. WHO. Obesity: preventing and managing the global epidemic. Report of a WHO consultation. World Health Organ Tech Rep Ser. 2000;894:i-xii, 1-253.

4. Yadav M, Akobeng AK, Thomas AG. Breast-feeding and childhood obesity. J Pediatr Gastroenterol Nutr. 2000 Mar;30(3):345-6. http://dx.doi.org/00005176200003000-00028

5. Pereira LO, Francischi RP, Lancha Jr AH. Obesidade: Hábitos Nutricionais, Sedentarismo e Resistência a Insulina. Arq Bras Endocrinol Metab. 2003;47(2):111-27. http://dx.doi.org/10.1590/S000427302003000200003

6. Foster-Schubert KE, Cummings DE. Emerging therapeutic strategies for obesity. Endocr Rev. 2006 Dec;27(7):779-93. http://dx.doi.org/er.2006-0041 [pii]10.1210/er.2006-0041

7. Tock L, Prado WL, Caranti DA, Cristofalo DM, Lederman H, Fisberg M, et al. Nonalcoholic fatty liver disease decrease in obese adolescents after multidisciplinary therapy. Eur J Gastroenterol Hepatol. 2006 Dec;18(12):1241-5. http://dx.doi.org/10.1097/01. meg.0000243872.86949.9500042737-20061200000001 [pii]

8. Reaven GM. Banting lecture 1988. Role of insulin resistance in human disease. Diabetes. 1988 Dec;37(12):1595-607. http://dx.doi.org/1595-607

9. Despres JP, Lemieux I. Abdominal obesity and metabolic syndrome. Nature. 2006 Dec;444(7121):881-7. http://dx.doi.org/nature05488 [pii]10.1038/nature05488

10. Oda E. The metabolic syndrome as a concept of adipose tissue disease. Hypertens Res. 2008 Jul;31(7):1283-91. http://dx.doi.org/JST.JSTAGE/hypres/31.1283 [pii]10.1291/hypres.31.1283

11. Volp AC, Alfenas RC, Costa NM, Minim VP, Stringueta PC, Bressan J. [Inflammation biomarkers capacity in predicting the metabolic syndrome]. Arq Bras Endocrinol Metabol. 2008 Apr;52(3):537-49. http:// dx.doi.org/S0004-27302008000300015 [pii]

12. Oda E. No existing definition of metabolic syndrome meets criteria as a syndrome. Int J Obes (Lond). 2008 Feb;32(2):393-4. http://dx.doi.org/10.1038/ sj.ijo.0803714

13. Galic S, Oakhill JS, Steinberg GR. Adipose tissue as an endocrine organ. Mol Cell Endocrinol. 2010 Mar;316(2):129-39. http://dx.doi.org/10.1016/j. mce.2009.08.018

14. Gregor MF, Hotamisligil GS. Inflammatory Mechanisms in Obesity. Annu Rev Immunol. 2011;29:41545. http://dx.doi.org/10.1146/annurev-immunol-031210-101322

15. Heber D. An integrative view of obesity. Am J Clin Nutr. 2010 Jan;91(1):280S-3S. http://dx.doi.org/ ajcn.2009.28473B [pii]10.3945/ajcn.2009.28473B

16. Tilg H, Moschen AR. Adipocytokines: mediators linking adipose tissue, inflammation and immunity. Nat Rev Immunol. 2006 Oct;6(10):772-83. http://dx.doi. org/nri1937 [pii]10.1038/nri1937

17. Arslan N, Erdur B, Aydin A. Hormones and cytokines in childhood obesity. Indian Pediatr. 2010 Oct;47(10):829-39. http://dx.doi.org/10.1007/s13312010-0142-y

18. Pedersen BK. The anti-inflammatory effect of exercise: its role in diabetes and cardiovascular disease control. Essays Biochem. 2006;42:105-17. http:// dx.doi.org/bse0420105 [pii]10.1042/bse0420105

19. Bradley RL, Jeon JY, Liu FF, Maratos-Flier E. Voluntary exercise improves insulin sensitivity and adipose tissue inflammation in diet-induced obese mice. Am J Physiol Endocrinol Metab. 2008 Sep;295(3):E586-94. http://dx.doi.org/00309.2007 [pii]10.1152/ajpendo.00309.2007

20. Winkler G, Kiss S, Keszthelyi L, Sapi Z, Ory I, Salamon F, et al. Expression of tumor necrosis factor (TNF)-alpha protein in the subcutaneous and visceral adipose tissue in correlation with adipocyte cell volume, serum TNF-alpha, soluble serum TNF-receptor-2 concentrations and C-peptide level. Eur J Endocrinol. 2003 Aug;149(2):129-35. http://dx.doi. org/10.1530/eje.0.1490129

21. Lyon CJ, Law RE, Hsueh WA. Minireview: adiposity, inflammation, and atherogenesis. Endocrinology. 2003 Jun;144(6):2195-200. http://dx.doi.org/10.1210/ en.2003-0285

22. Petersen AM, Pedersen BK. The anti-inflammatory effect of exercise. J Appl Physiol. 2005 Apr;98(4):1154-62. http://dx.doi.org/98/4/1154 [pii]10.1152/japplphysiol.00164.2004

23. Choi KM, Ryu OH, Lee KW, Kim HY, Seo JA, Kim SG, et al. Serum adiponectin, interleukin-10 levels and inflammatory markers in the metabolic syndrome. Diabetes Res Clin Pract. 2007 Feb;75(2):235-40. http:// dx.doi.org/S0168-8227(06)00285-3 [pii]10.1016/j. diabres.2006.06.019 
24. Nishida M, Moriyama T, Sugita Y, Yamauchi-Takihara K. Interleukin-10 associates with adiponectin predominantly in subjects with metabolic syndrome. Circ J. 2007 Aug;71(8):1234-8. http://dx.doi.org/JST. JSTAGE/circj/71.1234 [pii]

25. Batista Jr. ML, Lopes RD, Seelaender MC, Lopes AC. Anti-inflammatory effect of physical training in heart failure: role of TNF-alpha and IL-10. Arq Bras Cardiol. 2009 Dec;93(6):643-51, 92-700. http://dx.doi.org/ S0066-782X2009001200021 [pii]

26. Ropelle ER, Flores MB, Cintra DE, Rocha GZ, Pauli JR, Morari J, et al. IL-6 and IL-10 anti-inflammatory activity links exercise to hypothalamic insulin and leptin sensitivity through IKKbeta and ER stress inhibition. PLoS Biol. 2010;8(8):1-20. http://dx.doi. org/10.1371/journal.pbio.1000465

27. Ciolac EG, Guimarães GV. Exercício Físico e Síndrome Metabólica. Rev Bras Med Esporte. 2004;10(4):319-24. http://dx.doi.org/10.1590/S151786922004000400009

28. Donnelly JE, Blair SN, Jakicic JM, Manore MM, Rankin JW, Smith BK. American College of Sports Medicine Position Stand. Appropriate physical activity intervention strategies for weight loss and prevention of weight regain for adults. Med Sci Sports Exerc. 2009 Feb;41(2):459-71. http://dx.doi.org/10.1249/ MSS.0b013e3181949333

29. Gomez-Merino D, Drogou C, Guezennec CY, Chennaoui $\mathrm{M}$. Effects of chronic exercise on cytokine production in white adipose tissue and skeletal muscle of rats. Cytokine. 2007 Oct;40(1):23-9. http:// dx.doi.org/S1043-4666(07)00370-5 [pii]10.1016/j. cyto.2007.07.188
30. Corriveau P, Paquette A, Brochu M, Prud'homme D, Rabasa-Lhoret R, Lavoie JM. Resistance training prevents liver fat accumulation in ovariectomized rats. Maturitas. 2008 Mar 20;59(3):259-67. http:// dx.doi.org/S0378-5122(08)00044-3 [pii]10.1016/j. maturitas.2008.02.005

31. Pauli JR, Cintra DE, Souza CT, Ropelle ER. [New mechanisms by which physical exercise improves insulin resistance in the skeletal muscle]. Arq Bras Endocrinol Metabol. 2009 Jun;53(4):399-408. http:// dx.doi.org/S0004-27302009000400003 [pii]

32. Lira FS, Rosa JC, Pimentel GD, Tarini VA, Arida RM, Faloppa F, et al. Inflammation and adipose tissue: effects of progressive load training in rats. Lipids Health Dis. 2010;9:109. http://dx.doi.org/1476511X-9-109 [pii]10.1186/1476-511X-9-109

33. Leite RD, Prestes J, Bernardes CF, Shiguemoto GE, Pereira GB, Duarte JO, et al. Effects of ovariectomy and resistance training on lipid content in skeletal muscle, liver, and heart; fat depots; and lipid profile. Appl Physiol Nutr Metab. 2009 Dec;34(6):1079-86. http://dx.doi.org/h09-116 [pii]10.1139/h09-116.

34. Pighon A, Paquette A, Barsalani R, Chapados NA, Yasari S, Doucet E, et al. Substituting food restriction by resistance training prevents liver and body fat regain in ovariectomized rats. Climacteric. 2009 Apr;12(2):153-64. http://dx.doi.org/905698326 [pii]10.1080/13697130802447074

35. Speretta GF, Rosante MC, Duarte FO, Leite RD, Lino AD, Andre RA, et al. The effects of exercise modalities on adiposity in obese rats. Clinics (Sao Paulo). 2012 Dec;67(12):1469-77. http://dx.doi.org/S180759322012001200019 [pii]

Recebido: $\quad$ 19/08/2013.

Revisado: $\quad$ 22/11/2013.

Aprovado: $\quad$ 04/02/2014. 